Radial and Nonradial Pulsations as Probes of Stellar Physics

ASP Conference Series, Vol. 259, 2002

C. Aerts, T.R. Bedding, \& J. Christensen-Dalsgaard, eds.

\title{
The Influence of Dust Properties on the Mass Loss in Pulsating AGB Stars
}

\author{
Anja C. Andersen, Susanne Höfner, Rita Loidl \\ Department of Astronomy $\&$ Space Physics, Uppsala University, Box \\ 515, SE-751 20 Uppsala, Sweden
}

\begin{abstract}
We are currently studying carbon based dust types of relevance for carbon-rich AGB stars, to obtain a better understanding of the influence of the optical and chemical properties of the grains on the mass loss of the star. An investigation of the complex interplay between hydrodynamics, radiative transfer and chemistry has to be based on a better knowledge of the micro-physics of the relevant dust species.
\end{abstract}

\section{Introduction}

Asymptotic giant branch (AGB) stars show large amplitude pulsations with periods of about 100 to 1000 days. The pulsation creates strong shock waves in the stellar atmosphere, causing a levitation of the outer layers. This cool and relatively dense environment provides favorable conditions for the formation of molecules and dust grains. The dust formation basically determines the mass loss in these stars. Dust grains therefore play a very important role for the further evolution of the star (Sedlmayr, these proceedings).

Dust formation can take place only if (1) the temperature is sufficiently low, (2) the abundance of the dust forming species is sufficiently large and (3) the time scale providing favorable conditions is sufficiently long to allow for effective dust formation to proceed.

Condensation and evaporation of dust in envelopes of pulsating stars must be treated as a time-dependent process since the time scales for condensation and evaporation are comparable to variations of the thermodynamic conditions in the stellar atmosphere. The radiation pressure on newly formed dust grains can enhance excisting shock waves or even create shock waves leading to more or less pronounced discrete dust shells in the expanding circumstellar flow (e.g. Fleischer et al., 1992; Höfner \& Dorfi, 1997). We have calculated models of carbon-rich AGB stars with different carbon dust properties, in order to establish the dependence of the dynamical models on the material properties such as the opacity and the intrinsic density of the dust material.

\section{Carbon grains}

Amorphous carbon grains seem to be very good candidates for the most common type of dust particles present in circumstellar envelopes of carbon-rich AGB stars. 
Table 1. List of the different dust data shown in Fig. 1.

\begin{tabular}{|l|c|c|c|c|}
\hline Reference & $\begin{array}{c}\text { Material } \\
\text { name }\end{array}$ & $\begin{array}{c}\rho \\
\left(\mathrm{g} / \mathrm{cm}^{3}\right)\end{array}$ & $\begin{array}{c}\text { Designation } \\
\text { in this paper }\end{array}$ & Comments \\
\hline Jäger et al. (1998) & cel400 & 1.435 & Jäger 400 & "diamond-like" \\
Jäger et al. (1998) & cel1000 & 1.988 & Jäger 1000 & "graphite-like" \\
Maron (1990) & $\mathrm{AC} 2$ & 1.85 & Maron & $a$ \\
\hline
\end{tabular}

${ }^{a}$ Optical constants based on measurements by Bussoletti et al. (1987).

There exists a wide variety of possible amorphous carbon grain types, which fall in between the categories "diamond-like" and "graphite-like" amorphous carbon depending on the dominant type of chemical bonds. Different amorphous carbon dust data are listed in Table 1. The extinction efficiency data presented in this paper were calculated in the Rayleigh approximation for spheres (see Andersen et al. (1999) for details). As can be seen in Fig. 1a the difference in optical properties of different types of amorphous carbon is substantial.

\section{Dynamical models}

To obtain the structure of the stellar atmosphere and circumstellar envelope as a function of time we solve the coupled system of frequency-dependent radiation hydrodynamics and time-dependent dust formation (see Höfner, 1999 and Höfner et al., these proceedings for details). The dust formation is treated by the so-called moment method (Gail \& Sedlmayr, 1988; Gauger et al., 1990). In the moment method, dust formation is regarded as a two step process; (1) the formation of supercritical nuclei out of the gas phase and (2) the time dependent growth of grains to macroscopic sizes. The moment method is concerned with the time evolution of an ensemble of dust grains of various sizes and requires the nucleation rate as external input.

The models require as input the extinction efficiency $Q_{\text {ext }}$ of the grains ${ }^{1}$ and the intrinsic density of the material. The parameters of the models discussed here can be found in Table 2 . Wind properties like the mass loss rate $\dot{M}$, the time-averaged outflow velocity $\langle u\rangle$ and degree of condensation $\left\langle f_{\mathrm{c}}\right\rangle$ are direct results of the dynamical calculations. All elemental abundances are assumed to be solar except the one of carbon which is specified by an additional parameter, the carbon-to-oxygen ratio $\varepsilon_{\mathrm{C}} / \varepsilon_{\mathrm{O}}$.

\section{Results}

It is seen in Fig. 1b that the new models coincide reasonably well with observations of comparable stars. But at the same time it is clear from Table 2, that

\footnotetext{
${ }^{1}$ Or rather of the quantity $Q_{\text {ext }} / a$, which is independent of the grain radius, $a$, in the small particle (Rayleigh) limit which is applicable in this context.
} 

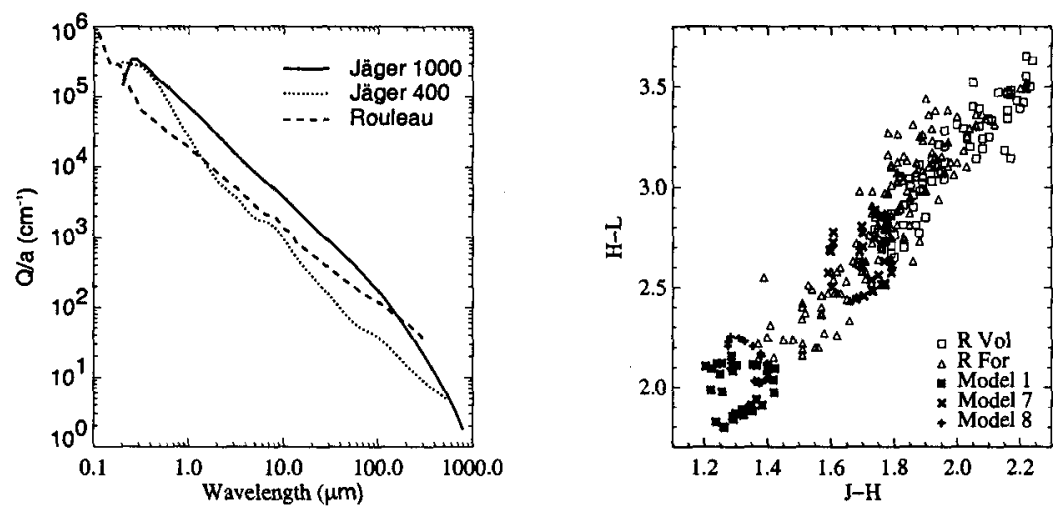

Figure 1. The figure (1a) to the left shows the extinction efficiency of amorphous carbon derived from optical constants (see Table 1 for annotations). The figure to the right $(1 \mathrm{~b})$ shows the $(\mathrm{H}-\mathrm{L})$ vs. $(\mathrm{J}-\mathrm{H})$ colors for two different models compared to observations of the bright Mira stars R Vol and R For which have moderate dust shell (Whitelock et al., 1997).

Table 2. Comparisc: of model results using different dust parameters. Model parameters: luminosity $L_{\star}\left(\right.$ in $\left.L_{\odot}\right)$, temperature $T_{\star}$ (in K), dust opacity data $\kappa_{\text {dust }}$, intrinsic dust density $\rho_{\text {dust }}$ (in $\mathrm{g} \mathrm{cm}^{-3}$ ), mass $M_{\star}=1.0 M_{\odot}$, carbon-to-oxygen ratio $\varepsilon_{\mathrm{C}} / \varepsilon_{\mathrm{O}}=1.4$, pulsation period $P=650 \mathrm{~d}$, velocity of the inner boundary $\Delta u_{\mathrm{p}}=4 \mathrm{~km} \mathrm{~s}^{-1}$. Results: mass loss rate $\dot{M}$ (in $\mathrm{M}_{\odot} / \mathrm{yr}$ ), mean velocity at the outer boundary $\langle u\rangle$ (in $\mathrm{km} / \mathrm{s}$ ), mean degree of condensation at the outer boundary $\left\langle f_{\mathrm{c}}\right\rangle$.

\begin{tabular}{|l|l|c|c||c|c|c|l|}
\hline $\begin{array}{l}L_{\star} \\
\left(\mathrm{L}_{\odot}\right)\end{array}$ & $\begin{array}{l}T_{\star} \\
(\mathrm{K})\end{array}$ & $\begin{array}{c}\kappa_{\text {dust }} \\
\left(\mathrm{cm}^{-1}\right)\end{array}$ & $\begin{array}{c}\rho_{\text {dust }} \\
\left(\mathrm{g} \mathrm{cm}^{-3}\right)\end{array}$ & $\begin{array}{c}M \\
\left(\mathrm{M}_{\odot}\right)\end{array}$ & $\begin{array}{c}\langle u\rangle \\
\left(\mathrm{m} \mathrm{s}^{-1}\right)\end{array}$ & $\left\langle f_{\mathrm{c}}\right\rangle$ & Comment \\
\hline 13000 & 2700 & Jäger1000 & 1.99 & $5.6 \cdot 10^{-6}$ & 15 & 0.05 & Model 1 \\
13000 & 2700 & Jäger1000 & $2.25^{*}$ & $7.3 \cdot 10^{-6}$ & 20 & 0.11 & Model 2 \\
13000 & 2700 & Rouleau & 1.85 & $4.3 \cdot 10^{-6}$ & 7 & 0.10 & Model 3 \\
13000 & 2700 & Rouleau & $2.25^{*}$ & $8.2 \cdot 10^{-6}$ & 18 & 0.31 & Model 4 \\
13000 & 2700 & Jäger400 & 1.44 & - & - & - & Model 5 \\
13000 & 2700 & Jäger400 & $2.25^{*}$ & $2.1 \cdot 10^{-8}$ & 1 & 0.13 & Model 6 \\
\hline 10000 & 2600 & Jäger1000 & 1.99 & $7.0 \cdot 10^{-6}$ & 16 & 0.09 & Model 7 \\
10000 & 2600 & Rouleau & 1.85 & $2.3 \cdot 10^{-6}$ & 4 & 0.12 & Model 8 \\
10000 & 2600 & Jäger400 & 1.44 & - & - & - & Model 9 \\
\hline
\end{tabular}

* Density of pure graphite. 
the mean outflow velocity, $\langle u\rangle$, and the degree of condensation, $\left\langle f_{\mathrm{c}}\right\rangle$, change significantly with the dust data used.

Comparing Model 1 and 3 the mean degree of condensation, $\left\langle f_{c}\right\rangle$, is much higher for the model using the dust data with the lower opacity, but at the same time the mean outflow velocity, $\langle u\rangle$, is higher for the model using the dust data with the higher opacity.

The degree of condensation also increases substantially if a higher intrinsic density for the material is assumed. In the models we have used both the true value of the material (Model $1,3,5,7,8,9$ ) as it was determined in the laboratory as well as the value of $\rho=2.25 \mathrm{~g} / \mathrm{cm}^{3}$, equivalent to the intrinsic density of pure graphite (Model 2, 4,6). The latter value has been used in many existing models (e.g. Fleischer et al., 1992; Höfner \& Dorfi, 1997). The result of using the higher density of graphite instead of the right value, is that the models become much redder since more dust is formed. Even a small increase of about $10 \%$ in the density of the dust material (as is the case from Model 1 to 2) results in a doubling of the degree of condensation and a substantial increase in the outflow velocity, $\langle u\rangle$. This stresses the importance of using the measured material value if possible, since another choice (even if it has been carefully considered) can create an artificial increase/decrease in the calculated mass loss of the models.

Acknowledgments. ACA greatfully acknowledges financial support from the Carlsberg Foundation. This work was supported by NorFA, the Royal Swedish Academy of Science and the Swedish Research Council.

\section{References}

Andersen, A.C., Loidl, R., \& Höfner, S. 1999, A\&A, 349, 243

Bussoletti, E., Colangeli, L., Borghesi, A., \& Orofino V. 1987, A\&AS, 70, 257

Fleischer, A.J., Gauger, A., \& Sedlmayr, E. 1992, A\&A, 266, 321

Gail, H.-P. \& Sedlmayr, E. 1988, A\&A, 206, 153

Gauger, A., Gail, H.-P., \& Sedlmayr, E. 1990, A\&A, 235, 345

Höfner, S. 1999, A\&A, 346, L9

Höfner, S. \& Dorfi, E.A. 1997, A\&A, 319, 648

Jäger, C., Mutschke, H., \& Henning, Th. 1998, A\&A, 332, 291

Rouleau, F. \& Martin, P.G. 1991, ApJ, 377, 526

Whitelock, P., Feast, M., Marang, F., \& Overbeek, M. 1997, MNRAS, 288, 512 